\title{
RITRATTO DI SIGNORA: LA SCIENZA AL FEMMINILE NELL'ICONOGRAFIA TRA SETTE E OTTOCENTO
}

\author{
PORTRAIT OF A LADY: WOMEN SCIENCE IN ICONOGRAPHY BETWEEN THE \\ 18TH AND 19TH CENTURY
}

Lucia De Frenza

Universidad de Bari, Italia

\section{Riassunto:}

Tra il Sette el'Ottocento era molto comune, per le donne interessate alla scienza, trovare nel matrimonio un'occasione per poter operare nel campo della ricerca insieme al marito. Questo è testimoniato dal valore mediatico dell'arte: grazie ai due ritratti dei coniugi Lavoisier e Galvani, è possibile comprendere il rapporto lavorativo, oltre che personale, all'interno della coppia.

\section{Palabras Claves:}

donna, Galvani, Lavoisier, scienza, ricerca, ritratto.

\section{Abstract:}

Between the 18th and 19th century it was very common, for those women interested in science, to find in marriage a chance to work in the research field along with their husbands. This is proved by media valour of the art: we can understand the relationship between husband and wife that went beyond the private field thanks to two portraits of the Galvanis and the Lavoisiers.

\section{KEY WORD:}

woman, Galvani, Lavoisier, science, research, portrait.. 
Donne, mogli di scienziati, nel Settecento: la presenza di una compagna nella vita di uomini consacrati all'investigazione della natura nell'età dei Lumi non riporta alla mente nei casi più felici soltanto l'immagine di una dedizione appassionata o di una tenerezza di affetti coniugali vissuti nel segreto delle mura domestiche; può, invece, alludere anche ad un legame di collaborazione, coltivato questo pure in privato, e ad una coincidenza di interessi ed intenti, che sarebbe da considerare come il primo nucleo di un'attività corale di ricerca.

In questo secolo per una donna appassionata di scienza l'essere maritata era una condizione che poteva volgersi a proprio vantaggio. In alcuni casi, come in quello della bolognese Laura Bassi (1711-1778), prima donna in Europa ad ottenere l'incarico ufficiale di lettore di Filosofia, il matrimonio accordò quella parvenza di legittimità, che le permise, senza scandalo, di occuparsi di fisica e in occasioni eccezionali di tenere lezioni accademiche agli uomini: da nubile, non avrebbe potuto sottrarsi al cliché che le era stato assegnato, di una Minerva rediviva, vergine votata allo studio ed alla castità, divina ed intoccabile.

In altricasi il vincolo coniugale costituì la condizione per far emergereed incrementare delle potenzialità intellettuali che altrimenti sarebbero state sacrificate. In genere non sembri, però, un'ovvietà - fanciulle dotate di grande intelligenza e amore per la conoscenza potevano mettere a frutto le loro doti, solo se trovavano nel proprio genitore il primo caloroso estimatore, che, oltre a provvederle di un'educazione conforme agli interessi ed alle capacità dimostrate, pur sempre esclusivamente domestica, cercavano di maritarle con giovani che possedevano le stesse ambizioni intellettuali. Questo fu il destino, per esempio, di Marie-Anne Paulze (1758-1836), che andò in sposa a tredici anni all'impiegato dell'esattoria generale, appassionato di chimica, Antoine Laurent Lavoisier $^{1}$ o di Lucia Galeazzi (1743-1790), figlia del bolognese professore di fisica e celebre anatomico, Domenico Gusmano, che accolse la richiesta di matrimonio del suo giovane assistente, Luigi Galvani, di famiglia modesta (il padre era un orefice), ma già apprezzato in ambito accademico. In entrambi i casi si trattò di un legame reso saldo dall'amore e dal rispetto reciproco, che creò la possibilità di un connubio, oltre che sul piano affettivo, anche su quello intellettuale e della ricerca scientifica. Questi casi non furono esclusivi, ma possono risultare paradigmatici.

1 L'attrattiva della giovane Marie-Anne era stata notata anche dal cinquantenne Conte d'Amerval che frequentava il salotto di famiglia. Nella Cornell's Lavoisier Collection si conserva una lettera di Monsieur Paulze, nella quale garbatamente respinge la proposta di matrimonio presentata dal conte. Come non accadeva comunemente, il padre rifiutò un ottimo partito, per venire incontro ai desider della figlia, che si aspettava avrebbe avuto maggiori prospettive di affermare la propria personalità e mettere a frutto quelle doti che erano ancora in erba, sposando un giovane di mezzi più modesti (un proprio dipendente), ma di grandi aspirazioni.
Le condizioni in cui si svolgeva generalmente l'attività investigativa nel Settecento - dentro le mura domestiche, con strumenti costruiti in proprio e assistenti occasional - rendeva possibile la collaborazione tra i coniugi non solo nelle attività correlate allo studio e all'esame delle teorie, ma anche in quelle più tecniche, come la sperimentazione in laboratorio. Per la maggior parte delle donne impegnate nella ricerca scientifica insieme ai membri della famiglia le remore e le costrizioni legate al proprio sesso, imposte dalle convenzioni sociali, diventavano meno rigide. Esse pagavano questa tolleranza con una minore autonomia, poiché l'oggetto dei propri interessi doveva coincidere con quello che veniva prescelto dal marito o dal padre e nei confronti di questi assumevano in gerere una condizione di dipendenza, che intaccava la loro autostima (Schiebinger 1989). Il contributo da esse apportato alla ricerca comune spesso restava nell'ombra o non si distingueva da quello del familiare più celebre. Anche quest'ultimo ometteva di riferirne nel resoconto che accompagnava l'esposizione dei risultati ottenuti concordemente, dal momento che l'atto di formalizzazione, richiesto dalla scrittura, ricadeva all'epoca sotto gli stessi condizionamenti morali e sessisti, che facevano apparire disdicevole per una donna la pratica della ricerca nel campo delle scienze naturali.

Era abbastanza frequente, per esempio, che l'autrice scegliesse l'anonimato per pubblicare le sue opere, a meno che il ricorso al proprio nome non dovesse servire a rimarcare l'eccezionalità della prestazione fornita. Nel caso di Lavoisier, non ci sono scritti o documenti che attestino la considerazione con cui il marito aveva accolto il supporto della moglie nelle ricerche di chimica; mentre Galvani era stato più esplicito nel tessere le lodi della propria compagna ed accennare all'aiuto da lei fornito. Nell'Elogio della moglie, dopo aver parlato dell'ottima educazione ricevuta da Lucia nelle Sacre Scritture, nella lingua italiana e tedesca, nella prosodia e nella storia Galvani dichiarava di affidarle i suoi lavori, perché li correggesse, "sicurissimo che nessun errore di tal genere sarebbe sfuggito nei suoi scritti alla sua coltissima moglie" (Galvani 1937, p. 13). In realtà, il ritratto che fornisce della compagna, purtroppo persa prematuramente, diventa agiografico ed improntato a far emergere soprattutto le sue virtù morali e muliebri. Diverse pagine sono dedicate alla descrizione delle pratiche di devozione, che era solita compiere con sincera pietà e riservatezza, all'elogio della modestia ed onestà che mostrava nelle occasioni mondane e all'amorevolezza e sollecitudine nei suoi confronti.

2 Galvani scrisse due elogi in latino per onorare la memoria dellamata Lucia: il primo, conservato tra i cimeli e i documenti di proprietà degli eredi, fu stampato nel 1937 a cura di P. Ferrarino. L'altro fu arrotolato ed inserito in un tubo di piombo, sepolto insieme al feretro di Lucia Galeazzi. Ritrovato in occasione della traslazione delle spoglie all'interno della chiesa del Corpus Domini di Bologna nel 1873, fu stampato nel 1938 nella traduzione di D. Zucchini. Galvani compose anche un epitaffio in morte di Lucia. 
Queste pagine parlano ancora di affetti privati. Se si volesse andare oltre la sfera personale e cercare elementi estranei allo scambio di intime premure tra i coniugi, le testimonianze di cui si avrebbe bisogno sarebbero sicuramente diverse. Si è scelto di richiamare il caso della coppia Lavoisier e di quella Galvani proprio per la possibilità di usare una di queste fonti collaterali, per così dire pubbliche, indirette. Si tratta dell'iconografia che appartiene a questi personaggi. Il valore mediatico dell'arte, cioé quello di trasporre in immagini un significato, consente in questo caso di ritagliare un'istantanea sulla relazione di vita e di lavoro dei coniugi Lavoisier e Galvani, che rispecchia un modo di percepire le loro storie in un certo senso più attinente al sentire collettivo o almeno a quello degli ambienti più vicini ai suddetti personaggi. Naturalmente in questo caso, perché il confronto sia in qualche modo significativo, occorre prescindere dal valore artistico o dalla resa pittorica dei ritratti, cercando di limitare l'esame alla lettura degli elementi narrativi contenuti nella rappresentazione. Infatti, il ritratto dei Lavoisier è molto più celebre dell'altro e fu eseguito quando i personaggi raffigurati erano ancora in vita ed all'apice del successo mondano e scientifico.

Realizzato da Jacques Louis David nel 1788 su commissione della famiglia Lavoisier, troneggiò nella sala da pranzo dell'abitazione privata della coppia e fu conservato dagli eredi fino al 1925, quando venne acquistato da Rockefeller e due anni dopo donato al Rockefeller Institute for Medical Research di New York. Nel 1977 grazie alla generosità di Wrightsman fu acquisito dal Metropolitan Museum of Art, dove attualmente è esposto (Poirier 1994). Nonostante la paternità illustre del dipinto, gli storici dell'arte hanno attribuito in genere scarsa considerazione alle implicazioni culturali e al significato mediatico veicolati dall'opera. Solo Mary Vidal (1995) e più recentemente Marco Beretta (2001) si sono posti l'obiettivo, in particolare, di chiarire come nella rappresentazione pittorica venga messa in risalto la natura del sodalizio scientifico tra i Lavoisier e quale ruolo sia assegnato alla compagna dell'ideatore della rivoluzione chimica. Non ci sono dubbi, infatti, che M.me Lavoisier col suo elegante abito bianco e la sua capigliatura vaporosa sovrasti la scena e sia la vera chiave di lettura di tutto il complesso plastico. A prima vista si potrebbe semplicemente scorgere nella posa sensuale della donna, che con la mano sinistra tocca delicatamente la spalla del marito, e nello sguardo rapito dell'uomo, seduto tra gli oggetti quotidiani della sua ricerca, una rappresentazione dell'intesa amorosa che legò i due coniugi e si riverberò in modo positivo sul lavoro scientifico realizzato dal padre della chimica moderna. Si tratta di un'immagine che ispira una mutua tenerezza ed una compenetrazione costruttiva un legame che fu per entrambi stimolo per l'esplicarsi della propria creatività. J.L. David frequentava il salotto di M.me Lavoisier e conosceva molto bene sia l'avvenente padrona di casa sia il gruppo di collaboratori, di cui la coppia si era attorniata e che condivideva la medesima ambizione di rifondare dalla base il sistema chimico antico.
All'artista certamente non sfuggiva che nel settembre del 1788 era stata stampata la traduzione di An essay on phlogiston di Richard Kirwan realizzata da Marie-Anne Lavoisier, ${ }^{3}$ con una sua prefazione e note dei Lavoisier, Guyton de Morveau, Laplace, Monge, Berthollet e Fourcroy. Nella corrispondenza che la donna scambiava con illustri studiosi anche stranieri era evidente come le sue cognizioni di chimica non fossero del tutto posticce, ma frutto di una preparazione accurata, dovuta alle lezioni del marito e di Jean-Baptiste Bucquet, e di una pratica quotidiana di discussione e, forse, verifica diretta. Il tono delle lettere è quello che ci si aspetterebbe tra interlocutori che si stimano a vicenda. Nella comunità scientifica, quindi, M.me Lavoisier era apprezzata come femme savante, senza che la locuzione avesse alcuna connotazione spregiativa. In questo ruolo la raffigurò anche David. Non si dimentichi che il quadro doveva dare ai frequentatori dell'abitazione dei Lavoisier un monito sul valore e le virtù dei loro proprietari: la ritrattistica all'epoca era usata come forma di esibizione borghese.

La donna pubblicò oltre la traduzione su menzionata, anche quella di un altro saggio di Kirwan, On the strengh of acids, per gli Annales de chimie, tredici tavole di illustrazioni per il Traité élémentaire de chimie di Antoine Lavoisier e la prefazione delle Mémoires de physique et de chimie dello stesso. Molti altri contributi, più che altro traduzioni e i giornali delle esperienze condotte all'Arsenale, restano tra i manoscritti conservati presso l' Académie des sciences di Parigi. ${ }^{4}$ In sintesi, Marie-Anne deve essere considerata con pari dignità tra i personaggi che fecero parte del gruppo di Lavoisier e a cui si attribuisce la rivoluzione nel campo della chimica settecentesca: in tale modo i contemporanei percepirono il suo ruolo accanto al marito.

Nel ritratto di David la presenza in primo piano della figura femminile rompe con il modello tradizionale, che vede la donna quale musa ispiratrice dell'uomo di genio. In effetti gli sguardi delle due figure nel ritratto non si incontrano: se Lavoisier volge gli occhi in sù a cercare il volto della moglie, quest'ultima sembra guardare al di fuori del ritratto, in direzione del pittore. In una triangolazione di questo tipo David avrebbe simbolicamente rappresentato il legame tra arte e scienza. É noto che MarieAnne Lavoisier fu dotata di un certo talento artistico, che affinò prendendo lezioni proprio da David (Badilescu 2001: 115-116 e Pinault-Sörensen 1994). Realizzò, oltre ad un autoritratto e ad alcuni disegni passati ai suoi eredi e solo recentemente attribuitile, anche le tavole per il Traité élémentaire de chimie pubblicato nel $1789 .{ }^{5}$ David allude a questa collaborazione dipingendo sul lato sinistro del quadro un raccoglitore di bozzetti

3 Il nome di Madame Lavoisier non fu inserito nel frontespizio dell'opera stampata a Parigi presso la tipografia Hôtel Serpente, così come non hanno paternità le poche note che furono aggiunte dalla donna.

4 La lista è consultabile attraverso il sito Web, Panopticon Lavoisier, realizzato da Marco Beretta (http://moro.imss.fi.it/lavoisier).

5 Contro la tendenza frequente per una donna a non apparire quale autrice, M.me Lavoisier firmò le tavole con il suo nome completo: "Paulse Lavoisier sculpsit". 
appoggiato su un leggio. Antoine Lavoisier è ritratto mentre con molta probabilità apporta le ultime correzioni all'opera di imminente pubblicazione, che segnerà una svolta nella storia della chimica: sopra il tavolo e per terra giacciono gli strumenti principali adoperati nelle esperienze e sul lato opposto si trovano le incisioni realizzate dalla moglie come corredo al testo. L'opera di Lavoisier, così come il ritratto di David, costituisce un esempio di connubio ben riuscito tra l'espressione artistica e la creatività scientifica. Il trait-d'union tra questi due mondi è rappresentato simbolicamente da M.me Lavoisier, realizzatrice di disegni e incisioni a carattere tecnico utilizzati come illustrazioni di saggi scientifici.

La presenza della giovane signora nel dipinto non ha soltanto questo significato. Il suo aspetto ricercato e voluttuoso cattura - come si è detto - gli sguardi sia dello scienziato che dell'artista e diviene in tal modo metafora della natura, l'oggetto che per entrambi è posto al centro della propria attività creativa.

Nella rappresentazione della femme savante David mostra di accondiscendere ai pregiudizi sessisti in voga all'epoca. M.me Lavoisier, in effetti, non è raffigurata all'opera, cioé al tavolo di lavoro in un atteggiamento paritario con l'illustre chimico, ma in piedi, accanto a lui, quindi in posizione ancillare e completamente dipendente. La sua opera si sostiene su quella del marito e ne è il complemento.

Dalla posa scelta dall'artista non viene chiarito se la donna ha avuto un ruolo concretamente attivo nel lavoro quotidiano di sperimentazione, come, invece, appare in due disegni, di pugno della stessa Marie-Anne, che la ritraggono seduta ad un banchetto, mentre registra su un diario l'andamento delle prove sperimentali. ${ }^{6} \mathrm{I}$ disegni in seppia (che presumibilmente dovevano servire come illustrazioni in qualche opera, ma poi non furono utilizzati) descrivono due esperimenti per lo studio della respirazione umana e mostrano un certo numero di ricercatori che cooperano, mettendo in atto una completa suddivisione dei compiti. M.me Lavoisier non si integra nel gruppo, ma resta in disparte ad osservare ciò che il marito orchestra e i suoi collaboratori eseguono. Kawashima ha evidenziato recentemente come questi due disegni siano le prime raffigurazioni in cui una donna appare inserita in un gruppo di ricerca (Kawashima 2005). Si tenga conto che nell'età dei Lumi, pur essendoci stata una grande apertura alla componente femminile in attività eminentemente virili come l'indagine scientifica, era molto raro che si potesse trovare un'esponente del gentil sesso impegnata nello stesso tempo nello studio e nella pratica sperimentale della ricerca. Sembra probabile che M.me Lavoisier abbia subito pur'essa il peso di questi condizionamenti e si sia

6 Nello stesso ruolo fu raffigurata M.me Lavoisier nel bassorilievo posto sotto il monumento pedestre di Lavoisier a Parigi, eretto nel 1900. Questo bassorilievo, evidentemente ispirato al disegno di Marie-Anne, ritrae il famoso chimico mentre esegue l'esperimento sulla combustione. Il blocco monumentale fu confiscato dai tedeschi durante la seconda guerra mondiale e andò disperso (Badilescu 2001: 116). lasciata assegnare dal gruppo un ruolo di certo non secondario, come è quello della registrazione degli eventi sperimentali, ma isolato e di natura diversa dagli altri.

Questo comportamento era anche una conseguenza del rispetto e dalla stima che la giovane nutriva per un marito talmente dotato. In sintesi, se M.me Lavoisier fu cooptata alla pari nel gruppo di ricerca che mise in atto una vera e propria rivoluzione ne campo della chimica, non riuscì completamente ad affrancarsi da quegli atteggiamenti antifemministi e settari che persistevano nella società del suo tempo. L'iconografia conserva tali retaggi, che appaiono anche e soprattutto come condizionamenti inconsci acquisiti dalle stesse ambiziose protagoniste della scienza.

Nel ritratto che rappresenta il primo esperimento di Galvani (la contrazione di una rana in concomitanza con l'emissione di una scintilla dalla macchina elettrica) tali problematiche appaiono smussate. Il dipinto, una tela ad olio realizzata dal pittore accademico Antonio Muzzi ${ }^{7}$ nel 1862, fa parte attualmente delle collezioni dei Musei universitari di Bologna ed è esposto nel Rettorato di quell'Ateneo. L'impostazione del quadro risente ovviamente dello stile artistico corrente e del modo di percepire e rappresentare quelle tendenze maturato dell'artista bolognese, la cui produzione si indirizzò per lo più a soddisfare commissioni locali. Rispetto a ciò che si nota nel capolavoro di David, qui i personaggi sono raffigurati in una posa meno ieratica, spiati in un momento di grande concentrazione, mentre sono tutti serrati intorno al tavolo dell'esperimento: essi hanno perso molto della compostezza e dell'armonia classica che contraddistingue le figure del pittore francese e che le rende simboli atemporali e modelli per gli osservatori. L'autore non ha scelto di rappresentare il dolce appagamento che segue un'attività creativa ormai quasi conclusa, ma l'attimo più entusiasmante della scoperta e della testimonianza corale. Sotto gli occhi dei suoi collaboratori, mentre un aiutante aziona la macchina elettrica, Galvani riesce a far agitare il corpo mutilato della rana. In primo piano, di fronte al marito, Lucia Galeazzi, senza gli orpelli consueti della sua condizione sociale, vestita semplicemente di verde scuro, guarda estasiata l'animale sul tavolo. Ma è questo che cattura tutta la scena: sotto una luce abbagliante, come un preparato sul vetrino di un microscopio, diviene l'emblema dell'oggetto moderno della scienza, l'elemento naturale modificato che risponde alle manipolazioni dello sperimentatore. Lucia è investita dalla luce che emana direttamente dall'animale, mentre si piega col busto in avanti per osservare più agevolmente il risultato dell'operazione.

7 Antonio Muzzi (1815-1894), nacque a Bologna, studiò e poi insegnò nella locale Accademia di Belle Arti. Fu un abile ritrattista, ma a lui si devono anche alcune pitture murali, come quelle realizzate per l'abitazione dei Malvezzi e Palazzo Facchini. Gli anni più creativi della sua esperienza artistica furono quelli tra il 1846 ed il ' 48 , quando si esercitò nella pittura, mettendo a frutto le tecniche e le idee maturate durante il recente soggiorno a San Pietroburgo. Alcune delle realizzazioni attribuitegli risentono della sua formazione accademica e restano affossate in un manierismo rococò arricchito con spunti neoclassici, mentre altre, soprattutto del genere storico, risultano più originali. 
Il dipinto fu realizzato nella seconda metà dell'Ottocento, poco prima dell'avvio delle celebrazioni galvaniane, volute dall'Università di Bologna per festeggiare il suo ottavo Centenario. Esso testimonia un rinnovato interesse non solo per una gloria cittadina, ma anche per un momento significativo della storia della scienza, che coincise con la nascita di una nuova disciplina, l'elettrofisiologia. Nell'interpretazione dell'opera di Galvani a metà dell'Ottocento erano emersi già molti stereotipi, che gli storici hanno ripreso per parecchi decenni ancora, rendendo difficile apprezzare le novità della sua proposta scientifica. Uno di questi stereotipi era stato voluto da una certa agiografia, che, sottovalutando l'opera dello scienziato, aveva, invece, posto l'accento sugli aspetti della sua esperienza personale, sugli ideali, le convinzioni religiose ed il carattere mostrato soprattutto nei tanti momenti difficili. Così era stato esaltato l'amore per la moglie Lucia, la giovane che gli aveva offerto la propria comprensione e lo aveva assistito nelle attività di ricerca. ${ }^{8}$ Come avevano riportato alcune fonti coeve, la donna era stata una frequentatrice del laboratorio privato come aiutante (Galvani aveva prediletto per questo compito i membri della propria famiglia e pochi amici fidati, come il gesuita Rialp). La tradizione riporta anche che ad accorgersi della contrazione della rana nel cosidetto "primo esperimento" di Galvani era stata Lucia, la quale, giudicandolo un fatto nuovo, aveva avvertito il marito, assente in quel momento, e lo aveva invitato a ripetere l'esperimento. Questo, per esempio, era il racconto riportato da Alibert nell’Elogio storico di Luigi Galvani (1802). Alla sposa di Galvani, "questa incomparabile donna" (p. 36), Alibert attribuiva la sagacità di aver intuito che vi era un collegamento tra la manipolazione della rana con uno scalpello e la scintilla scoccata contemporaneamente dalla macchina elettrica collocata nella stessa stanza. Ripetuto l'esperimento da Galvani il risultato fu identico. Questo momento è raffigurato da Muzzi nel suo ritratto. Probabilmente la luce che dalla rana irradia il volto di Lucia è usata per sottolineare la brillante intuizione attribuita alla donna, da cui derivò una scoperta tanto importante. In realtà non esistono attestazioni che costituiscano una prova certa di questo evento. Galvani, quando nel De viribus riferì il racconto della scoperta, non nominò gli aiutanti che erano con lui in quell'occasione (Galvani 1791: p. 364). La notizia data da Alibert trovava conferma in un sonetto che circolò a Bologna subito dopo la pubblicazione del saggio di Galvani, la cui seconda terzina alludeva al modo in cui Lucia, toccando con lo scalpello i nervi dell'animale, "novo ardor vitale in rana ignuda a disvelar pur giunse". Ma le notizie riportate da Alibert devono essere prese col beneficio del dubbio; tant'è vero che lo stesso giustifica la presenza di rane

8 A Lucia Galeazzi non è attribuita alcuna opera, né traduzione, né altro. Interpreti più recenti, come Piccolino e Bresadola (2003) hanno sottolineato la convenienza del matrimonio con Lucia per il giovane ed ambizioso neo laureato, che grazie all'intervento del suocero riuscì in pochi anni a scalare tutti i gradini della carriera accademica. Le ricerche di Galvani negli anni in cui visse Domenico Gusmano Galeazzi coincisero con quelle del vecchio professore. Non si dimentichi che l'accordo matrimoniale prevedeva che gli sposi abitassero insieme ai suoceri e non è difficile immaginare che Galvani potesse utilizzare anche lo studio e gli strumenti che appartenevano al padrone di casa. quel giorno nel laboratorio di Galvani con la sua intenzione di preparare un brodetto per la moglie cagionevole di salute. D'altro canto in alcuni manoscritti che riguardano le ricerche eseguite nel 1781 Galvani sembra alludere all'aiuto ricevuto da Lucia (Gherardi 1868, p. 11).

Per chiarire il tipo di coinvolgimento che la donna ebbe nelle ricerche del marito occorre certamente un'analisi più approfondita delle fonti, che finora non è stata compiuta. L'artista che l'ha dipinta al tavolo di lavoro di Galvani insieme agli altri collaboratori non ha avuto, invece, dubbi: si può vedere in questo quadro una scena insolita nella raffigurazione dello scienziato all'opera (soprattutto che ci si riferisce ad un personaggio del Settecento), perché la figura femminile ritratta non rappresenta né la musa, né una spettatrice ignara, ma un componente a tutti gli effetti del gruppo di ricerca. L'iconografia sembra in questo caso risolvere un problema storico ancora aperto. Più che attribuire all'artista una mancanza di criticità, occorre vedere la sua opera come lo specchio di un'interpretazione romanticizzata del fatto storico. L'arte mostra di avere anche libertà di invenzione: è questa una sua prerogativa essenziale. ${ }^{9}$

\section{RIFERIMENTI BIBLIOGRAFICI}

Alibert, J-L.-M., Elogio storico di Luigi Galvani, Bologna, S.T. d'Aquino, 1802.

Badilescu, S., "Chemistry for Beginners. Women Authors and Illustrators of Early Chemistry Textbooks", Chemical Educator, 6 pp. 114-120, 2001.

Beretta, M., Imaging a Career in Science. The Iconograpgy of Antoine Laurent Lavoisier, Richini, Milano, 1748.

Bernabeo, R., Galeazzi Domenico Maria Gusmano: vita ed opere, Fermo, Tip. sociale, 1963.

Biagi Maino, D., “L'immagine dello scienziato, Atti della XVII edizione delle Giornate dell'Osservanza su Luigi Galvani (1737-1798), 9-10 maggio 1998", Quaderni 3, pp. 7593, Città di Castello, 1998.

Biagi Maino, D., Maino, G., "Luigi Galvani: lo scienziato oltre l'immagine", in Accademia delle Scienze dell'Istituto di Bologna, Discorsi e scritti in onore di Luigi Galvani nel bicentenario della morte 1798-1998, Sala Bolognese, Forni, pp. 155-167, 1999.

Bresadola, M., Pancaldi, G. (Eds.), Luigi Galvani international Workshop: Proceedings: Bologna, 9 october 1998, Università di Bologna, Bologna, 1999.

Carlone, L., Lucia Galeazzi Galvani "Negli Elogi" di Luigi Galvani, in Accademia delle Scienze dell'Istituto di Bologna, Discorsi e scritti in onore di Luigi Galvani nel bicentenario della morte 1798-1998, Sala Bolognese, Forni, 1999, pp. 143-148, 1999.

9 Il confronto tra il tavolo di laboratorio come è rappresentato nelle incisioni che corredano il saggio di Galvani e quello dipinto da Muzzi mostra quanto la rappresentazione possa essere diversa seconda degli scopi che si prefigge. 
Donovan, A., Lavoisier and David. Science, Art and the Revolution, in Lavoisier i els orígens de la química moderna, 200 anys després (1794-1994), Barcellona, Societat Catalana d'Història de la ciència i de la tècnica, pp. 132-145, 1996.

Galvani, L., "De viribus electricitatis in motu musculari commentarius", in De Bononiens Scientiarum at Ertium Instituti atque Academiae Commentarii, 7, pp. 363-418, 1791.

----, Elogio della moglie Lucia Galeazzi Galvani: testo latino con la traduzione italiana [di Pietro Ferrandino], Bologna, Tip. Azzoguidi, 1937.

----, Elogio dettato da Luigi Galvani in memoria della moglie Lucia Galeazzi: testo tratto dalla pergamena deposta dal marito nella tomba della moglie pubblicato per la prima volta da Dino Zucchini, Tip. L. Parma, Bologna, 1938.

----, Opere scelte, a cura di Barbensi G., Utet, Torino, 1967.

Gherardi, S., "Le illustrazioni di tre distinti manoscritti", Memorie dell'Accademia delle Scienze di Bologna, S. II, 81868.

Gottarelli, E., Il pittore accademico Antonio Muzzi a Bologna e a Pietroburgo, Tip. L. Parma, Bologna, 1979.

Kawashima, K., "Madame du Châtelet et Madame Lavoisier, deux femmes de science", in La Revue du Musée des Arts et Métiers, 22 pp. 22-29, 1998.

---, "Madame Lavoisier et la traduction française de l'Essay on phlogiston de Kirwan", in Revue d'Histoire des Sciences, 53-2, pp. 235-263, 2000.

Kawashima, K., "Madame Lavoisier. Participation of a Salonière in the Chemical Revolution", in Beretta M. (Ed.), in Lavoisier in Perspective, Munchen, pp. 79-94, Deutusches Museum, 2005.

Medici, M., Compendio storico della scuola anatomica di Bologna dal Rinascimento a tutto il secolo XVIII, Tipografia Governativa Della Volpe e del Sassi, Bologna, 1857.

Mesini, C., Luigi Galvani, Tip. S. Francesco, Bologna, 1958.

Mesini, C., Nuove ricerche galvaniane, Tamari, Bologna, 1971.

Ministero per i Beni e le Attività Culturali, Fondazione del Monte di Bologna e Ravenna, Antonio Muzzi: la fatica della creazione (Bologna, 1815-1894), Editrice Compositori, Pinacoteca Nazionale, Bologna, 1999.

Pera, M., La rana ambigua: la controversia sull'elettricità animale tra Galvani e Volta, Einaudi, Torino, 1986

Piccolino, M., Bresadola, M., Rane, torpedini e scintille. Galvani, Volta e l'elettricità animale, Bollati Boringhieri, Torino, 2003.

Pinault Sorensen, M., "Madame Lavoisier, dessinatrice et peintre", La Revue du Musée des Arts et Métiers, 6 (pp. 23-5, 1994
Poirier, J.-P., "Le couple Lavoisier sous l'oeil de David", La Revue du Musée des Arts et Métiers, 6 pp. 26-29, 1994.

----, Histoire des femmes de science en France, Paris, Pygmalion, 2002.

----, La science et l'amour. Madame Lavoisier, Paris, Pygmalion, 2004.

Rayner-Canham, G., Rayner-Canham, M., Women in chemistry, Chemical Heritage Foundation, Philadelphia, 2001.

Schiebinger, L., The mind has no sex?, Cambridge (Mass.), Harvard Univ. Press, 1989.

Smeaton, W. A., "Monsieur and Madame Lavoisier in 1789: The Chemical Revolution and the French Revolution", Ambix, 36, pp. 1-4, 1989.

Vidal, M., "David among the moderns: Art, Science, and the Lavoisier", Journal of the History of Ideas, 56, pp. 595-623, 1995.

Zucchini, G., Saggio di iconografia galvaniana: monumenti, ritratti, medaglie, luoghi, L. Cappelli, Bologna, 1937. 